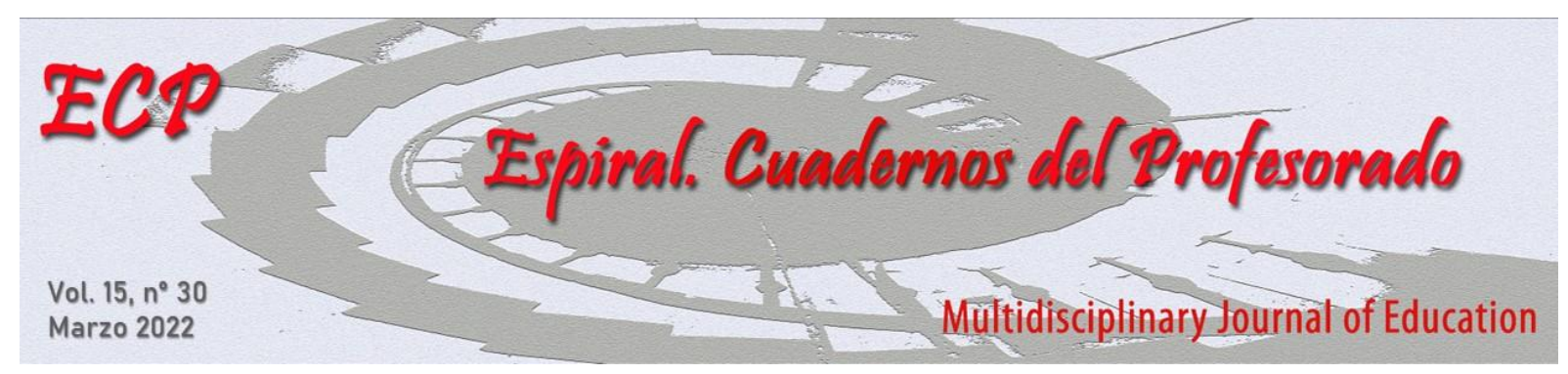

\title{
Relación entre la intención de ser físicamente activo y el desplazamiento al centro educativo: propuestas de intervención para potenciar el desplazamiento activo en niños
}

\section{Relationship between the intention to be physically active and active commuting to school: proposals for intervention to increase active commuting in children}

\author{
David Cerro-Herrero ${ }^{1}$, Josué Prieto-Prieto ${ }^{2}$, Miguel Angel Tapia Serrano ${ }^{1}$, Mikel Vaquero- \\ Solís y Pedro Antonio Sánchez-Miguel ${ }^{1}$ \\ ${ }^{1}$ Universidad de Extremadura, España; ${ }^{2}$ Universidad de Salamanca
}

\begin{abstract}
Resumen
El presente estudio analiza el desplazamiento activo al colegio en escolares de primaria y su posible relación con la intención de ser físicamente activo. Un total de 203 estudiantes ( 6 a 12 años) contestaron un cuestionario sobre hábitos de desplazamiento semanal al colegio y sobre su intención de ser físicamente activos. Aproximadamente la mitad de los participantes se desplazan de forma activa al colegio (50.8\%), no se encontraron diferencias significativas por sexo, ni por edad respecto a la forma de desplazarse, pero sí se hallaron diferencias en función de la distancia entre el domicilio y el centro educativo, siendo los que viven cerca quienes suelen desplazarse de forma más activa. En lo referente a la intención de ser físicamente activo se obtuvo una puntuación media de 4.37 (DT $=0.6 \%$ ) sobre un máximo de 5, pero no se encontró relación con el modo de desplazamiento activo. Es necesario implementar intervenciones que fomenten la visión del desplazamiento activo como una actividad físicodeportiva (mediante la inclusión de elementos de competición, cooperación, gamificación, retos y visibilidad) para poder de este modo aprovechar los altos niveles de intención de ser físicamente activo para aumentar los niveles de desplazamiento activo en los escolares e intentar de este modo superar la barrera que supone vivir lejos del centro educativo.
\end{abstract}

Palabras clave: Niños; escolares; actividad física; motivación

Abstract

The study analyzes active commuting to school in primary school children and its possible relationship with the intention of being physically active. A total of 203 students (6 to 12 years old) answered a questionnaire about weekly commuting habits to school and about their intention to be physically active. Approximately half of the participants actively travel to school $(50.8 \%)$, no significant differences were found by sex or age regarding the way of moving, whilst differences were found according to the distance between home and school, with those living close by tending to be more active commuters. Regarding the intention to be physically active, an average score of $4.37(\mathrm{Dt}=0.6 \%)$ was obtained out of a maximum of 5, but no relationship was found regarding the mode of active shifting. It is necessary to implement interventions that promote the vision of active shifting as a physicalsports activity (by including elements of competition, cooperation, gamification, challenges and visibility) in order to take advantage of the high levels of intention to be physically active in order to increase levels of active commuting in school children.

Keywords: Children; school; physical activity; motivation

Fecha de recepción: 20/07/2021

Fecha de aceptación: 06/10/2021

Correspondencia: Miguel Ángel Tapia Serrano, Universidad de Extremadura, España Email:matapiase@unex.es 


\section{Introducción}

Los sujetos con patrones habituales de actividad física (AF, de aquí en adelante) tienen menos probabilidad de desarrollar problemas de salud, por ello es innegable la necesidad de realizar AF de forma sistemática para tener una buena salud (Rosa-Guillamón, 2019). Por lo tanto, es importante crear oportunidades tanto dentro como fuera del contexto escolar, que permitan el aumento de los niveles de AF de niños y adolescentes para favorecer el estado de salud integral (Escobar-Gómez et al., 2020). El desplazamiento activo al y desde el centro educativo, consiste en desplazarse de forma no motorizada (caminando, en bicicleta, en/con patines o skate, entre otros) y se presenta como una oportunidad para aumentar el nivel de actividad física tanto en niños como en adolescentes (García-Hermoso et al., 2017).

La escuela ofrece diversas oportunidades para una actividad física diaria al margen de las clases de educación física, como los tiempos de recreo o al inicio y final de la jornada escolar. El profesorado debe conocer estas posibilidades y contar con recursos para potenciarlas (Cerro et al., 2021). Por todo ello el desplazamiento activo está siendo objeto de numerosos estudios para comprenderlo y poder potenciarlo en los centros educativos.

En los últimos años se ha intentado demostrar la relación entre el desplazamiento activo al centro escolar, los niveles de condición física y la cantidad de AF diaria acumulada. Henriques-Neto et al. (2020) en su revisión sistemática de 16 estudios a nivel mundial no encontraron relación consistente entre desplazarse activamente y los niveles de condición física en jóvenes. Algunos estudios han encontrado que los estudiantes que van de forma activa al centro educativo son más activos en su día a día (Larouche et al., 2014) pero, por otro lado, Garrote-García (2016) no encontró relación entre el desplazamiento activo y los niveles de actividad física en jóvenes. Incluso algunos estudios han encontrado que los estudiantes que van de forma activa al colegio son menos activos y participan menos en actividades deportivas (Mann et al., 2018).

Ante la disparidad de resultados en las investigaciones es necesario profundizar sobre la relación entre el desplazamiento activo y los comportamientos saludables. Diversos estudios han demostrado que la intención de ser físicamente activo es un predictor de los niveles de actividad física en niños y jóvenes. Martínez-Baena (2016) comprobó que la intención de ser físicamente activo predice el nivel de Actividad Física de Moderada a Vigorosa (AFMV, de aquí en adelante), To et al. (2020) al estudiar escolares de primaria descubrieron una asociación significativa entre la intención de ser físicamente activo y el número de pasos diarios, en la misma línea Mayorga-Vega et al., (2019) encontraron diferencias entre chicos y chicas en la intención de ser físicamente activos y en los niveles de AFMV, actividad física total, así como en los porcentajes que cumplían las recomendaciones de 10.000 pasos diarios. Por tanto, la intención de ser activo se ha mostrado como uno de los predictores más destacados de la actividad física en estudios realizados con niños y adolescentes (Kamtsios, 2011).

El presente estudio se plantea como objetivo analizar si existe una asociación entre el desplazamiento activo al centro educativo y la intención de ser físicamente activo. A este respecto se planteó como hipótesis que la intención de ser físicamente activo se relacionara de forma positiva con los niveles de desplazamiento activo de los escolares.

\section{Metodología}

\section{Diseño del estudio y participantes}

El estudio presenta un diseño transversal descriptivo y de tipo relacional. Participaron 203 escolares españoles, 102 niños (50.2\%) y 101 niñas (49.8\%) cursando Educación Primaria de la ciudad de Cáceres (muestra de conveniencia). Las edades estaban comprendidas entre los 6 y 12 años (alumnos de $1^{\circ}$ y $6^{\circ}$ curso $)$, siendo la edad media $(M=9.96 ; D T=1.57)$. La selección de la muestra fue realizada por un muestreo aleatorio intencional según la disponibilidad de los centros para colaborar, y la disponibilidad del investigador para desplazarse a los centros con el fin de realizar la recogida de datos. 


\section{Instrumentos}

Intención de ser físicamente activo. Se empleó el cuestionario Medida de la Intencionalidad para ser Físicamente Activo (MIFA), creado por Hein et al. (2004) y adaptado al contexto español por Moreno et al., (2007) con una muestra de adolescentes españoles. Además, es un instrumento que ha sido empleado con escolares de educación primaria españoles recientemente (Pérez-Soto et al., 2019). Arias et al., (2013), analizaron sus propiedades psicométricas con estudiantes de educación primaria y el instrumento mostró una alta consistencia interna $(\alpha=.80)$ y fiabilidad temporal $(\mathrm{ICC}=.79)$. Para este este estudio el valor del alpha de Cronbach $(\alpha)$ para la intención de ser físicamente activo es de 0.71. Se trata de un cuestionario ampliamente utilizado en escolares que consta de diversas afirmaciones, y que deben ser respondidas haciendo uso de una escala tipo Likert que oscila desde 1 (nada) a 5 (mucho). A partir de la puntuación obtenida se clasificó a los participantes en función de su intención de ser físicamente activos en: nada (promedio de 1 a 1.99 puntos), poco (promedio de 2 a 2.99 puntos), bastante (promedio de 3 a 3.99 puntos) y mucho (promedio de 4 puntos o más).

El desplazamiento activo se obtuvo mediante la realización del cuestionario auto-reportado PACO (Pedalea y Anda al Cole) de la Universidad de Granada. Este instrumento consta de cuatro ítems respecto al modo del desplazamiento: ¿cómo vas? ¿cómo vuelves? Para cada día de la semana, y al tiempo y la distancia de dicho desplazamiento, así como un ítem sobre intención futura de forma de desplazarse (Chillón et al., 2017). A partir de dicho cuestionario se estableció como activos aquellos alumnos con más 8 viajes activos a la semana siguiendo el estudio de Herrador-Colmenero et al., (2019). Se generó una variable identificando como estudiantes que viven cerca a aquellos que la distancia entre el centro educativo y su domicilio es menor de $1.5 \mathrm{~km}$ y lejanos a los que tienen que recorrer mayor distancia.

\section{Procedimiento}

El presente estudio se ha desarrollado siguiendo las siguientes fases:

1.- En primer lugar, se realizó una lista de los posibles colegios de la ciudad de Cáceres que podrían estar abiertos a participar en la investigación.

2.- Posteriormente se concertó una cita con el equipo directivo para explicar cuál sería el objeto de estudio y que no se vería comprometida la identidad de los participantes.

3.-Una vez obtenido el permiso de los centros educativos se procedió a entregar un consentimiento informado para solicitar el permiso y aprobación de los padres, explicando el objetivo del estudio y las variables a valorar.

El estudio fue previamente aprobado por el comité ético de la universidad de Extremadura, con el código "145/2019". Todos los participantes fueron tratados de acuerdo a los principios éticos y códigos de conductas de la American Psychological Association (2010) para este tipo de investigaciones. La prueba consistió en cumplimentar un cuestionario en unos 10 minutos aproximadamente durante la clase de educación física, en el cual el investigador principal estaba presente por si surgía alguna duda.

\section{Análisis Estadístico}

Se han realizado pruebas de estadística descriptiva, incluyendo medias y desviación típicas, empleando tablas de contingencia con prueba de Chi - cuadrado, para determinar la relación entre las variables. Se realizó el Test de Kolmogorov-Smirnov para observar la distribución normal de las variables $\mathrm{n}^{\mathrm{o}}$ de desplazamientos activos y puntuaciones en MIFA, obteniendo $\mathrm{p}<0.05$, y por tanto encontrándonos antes una distribución no normal. Se estudió la relación entre la puntuación obtenida en el cuestionario MIFA y las variables sociodemográficas y de desplazamiento activo. Se aplicaron las pruebas no paramétricas, U de Mann -Whitney. Se utilizó software SPSS 23.0.

\section{Resultados}

En primer lugar se realizó un análisis descriptivo mediante tablas de contingencias en cuanto a la movilidad al centro educativo, de lo cual se pudo observar que un $32.5 \%$ de los participantes no realizaban ningún viaje activo a la semana y un $49.3 \%$ realizaban menos de 8 viajes activos a la semana y por tanto fueron considerados pasivos en cuanto al desplazamiento activo, mientras que el 50.8\% de los participantes 
realizan 8 o más viajes a la semana de forma activa (caminando o en bicicleta) y por tanto fueron considerados activos. Respecto a los elementos que pueden condicionar el desplazamiento al centro educativo se observó que el $64.5 \%$ ( $n=131)$ viven a menos de 1.5 kilómetros del centro y el $35.5 \%(n=72)$ viven a mayor distancia. Destacando que el $81.8 \%$ tarda menos de 15 minutos en llegar al centro educativo desde su domicilio. En lo que se refiere al acompañamiento el $87.7 \%(n=178)$ realiza el trayecto de ida y/o vuelta acompañada por adultos, el $4.9 \%(n=10)$ lo hace en compañía de otros niños/as y el $7.4 \%(n=15)$ lo hace en solitario. Respecto a la forma de desplazamiento deseada por los escolares destaca que un $65.4 \%$ le gustaría hacerlo de forma activa ( $23.6 \%$ andando, $30 \%$ en bicicleta y $11.8 \%$ en patinete).

En lo referente a la intención de ser físicamente activo se obtuvo una puntuación media de 4.37 $(D T=.6 \%)$ sobre un máximo de 5 . No existe una asociación estadísticamente significativa entre la intención de ser físicamente activo y el sexo al realizar la prueba de Chi-Cuadrado $\left(\chi^{2}(2)=1.95, p=.907\right)$. Tampoco entre la intención de ser físicamente activo y el modo de desplazamiento $\left.\chi^{2}(2)=1.756, p=.416\right)$.

No se identificaron diferencias en la intención de ser físicamente activo según el sexo $(Z=-.413$; $p=.679$; prueba Mann-Whitney), ni por el tipo de desplazamiento al colegio $(\mathrm{Z}=-.593 ; \mathrm{p}=.553$; prueba Mann-Whitney) (Tabla 1).

Tabla 1.

Medida de la intencionalidad de ser físicamente activo por sexo y tipo de desplazamiento.

\begin{tabular}{llllll}
\hline MIFA & Total \% (n) & Chicos \% (n) & Chicas \% (n) & Activos \% (n) & Pasivos \% (n) \\
\hline Nada & $0(0)$ & $0(0)$ & $0(0)$ & $0(0)$ & $0(0)$ \\
Poco & $2.5(5)$ & $2.9(3)$ & $2.0(2)$ & $3.9(4)$ & $1.0(1)$ \\
Bastante & $17.7(36)$ & $17.6(18)$ & $17.8(18)$ & $17.5(18)$ & $18.0(18)$ \\
Mucho & $79.8(162)$ & $79.4(81)$ & $80.2(81)$ & $78.6(81)$ & $81.0(81)$ \\
\hline
\end{tabular}

Al analizar la asociación ente la distancia entre el domicilio y el centro educativo y el modo de desplazarse (Tabla 2), se encontró mediante la prueba de Chi-cuadrado una asociación estadísticamente significativa, siendo aquellos que viven más cerca los que optan por desplazamientos activo de forma más habitual $\left(\chi^{2}(2)=56.50, p<.005\right)$.

Tabla 2.

Tipo de desplazamiento al centro educativo en función de la distancia a la que vive el alumnado.

\begin{tabular}{llll}
\hline Tipo de desplazamiento & Total \% (n) & Cerca \% (n) & Lejos \% (n) \\
\hline Activo & $68.8 \%(95)$ & $69.8(95)$ & $7.8(8)$ \\
Pasivo & $35.5(43)$ & $31.2(43)$ & $87.7(57)$ \\
\hline
\end{tabular}

Finalmente se analizó la relación entre la edad y la forma actual de desplazarse y la intención futura de forma de desplazamiento (Tabla 3). Sin encontrarse relaciones significativas entre la edad y el desplazamiento actual o la intención de desplazamiento.

Tabla 3.

Modo de desplazamiento actual y futuro según la edad.

\begin{tabular}{lllll}
\hline \multirow{2}{*}{ Grupo de edad } & \multicolumn{2}{l}{ Desplazamiento actual } & \multicolumn{2}{l}{ Desplazamiento deseado } \\
\cline { 2 - 5 } & Activo \% (n) & Pasivo \% (n) & Activo \% (n) & Pasivo \% (n) \\
\hline 6-7 años & $57.7(15)$ & $42.3(11)$ & $57.7(15)$ & $42.3(11)$ \\
8-9 años & $34.8(24)$ & $65.2(45)$ & $65.2(45)$ & $34.8(24)$ \\
10-11 años & $60.4(55)$ & $39.6(36)$ & $65.9(60)$ & $34.1(31)$ \\
12 o más años & $52.9(9)$ & $47.1(8)$ & $76.5(13)$ & $23.5(4)$ \\
\hline Chi-Cuadrado & $\chi^{2}(2)=10.991, p=.012$ & $\chi^{2}(2)=1.617, p=.655$ \\
\hline
\end{tabular}




\section{Discusión}

La intencionalidad de ser activo en jóvenes ha sido estudiada como un factor determinante para la realización de práctica físico-deportiva real en el periodo extraescolar (Pérez-Soto et al., 2019). En la muestra del estudio se han obtenido valores superiores a estudios previos en cuanto a intención de ser físicamente activo, un 97.5\% tienen bastante o mucha intención de ser físicamente activo. Todos los estudios previos encontraron diferencias significativas por sexo, siendo los varones los que tenían mayores intenciones (Pérez-Soto et al., 2019; Sanz et al., 2017), pero en los escolares cacereños estudiados en esta investigación no se hallaron diferencias por sexo.

Estudios previos han corroborado la relación entre la intencionalidad de ser activo y la actividad física habitual autoinformada en escolares de etapa primaria (Kamtsios, 2011; Pérez-Soto et al., 2019), pero el presente estudio no encontró relación entre la intencionalidad de ser físicamente activo y el desplazamiento activo al centro educativo. Siendo la barrera de distancia al centro educativo la cual mantenía una relación con el tipo de desplazamiento, de modo que aquellos alumnos que vivían a más de $1.5 \mathrm{~km}$ tenían muchas menos posibilidades de optar por un desplazamiento activo (en bicicleta o caminando), como ya se describió en estudios previos sobre barreras para el desplazamiento activo (Cerro et al., 2020; Molina-García et al., 2016).

En lo que se refiere a la influencia de la edad en el modo de desplazarse o el modo deseado de desplazamiento no se han encontrado asociaciones significativas, pero en términos generales se observa que los alumnos de más de 10 años suelen ser más activos en el desplazamiento actual y a partir de los 8 años se muestra un mayor interés por el desplazamiento activo. Lo cual va en línea con resultados obtenidos en algunos estudios previos que analizan niños y adolescentes (Rodríguez-López, 2017).

Una vez analizados los datos se plantea que es necesario desarrollar ciertas intervenciones para que los estudiantes perciban el desplazamiento activo como una actividad física, ya que, la falta de relación entre la intención de ser físicamente activo y esta práctica cotidiana, es posible que se deba a la percepción del desplazamiento como una rutina no relacionada con la actividad física. Por todo ello se plantea la necesidad de integrar en el desplazamiento activo los valores de competición, gamificación, cooperación, retos y visibilidad para potenciar el desplazamiento activo como práctica físico-deportiva. En este sentido son varias las investigaciones que han mostrado bueno resultados asociando el desplazamiento activo por ejemplo con la competición, por ejemplo en España, De la Cruz Bazaga et al., (2021) promoviendo una competición escolar para sumar kilómetros tanto de forma individual como por clases o en Reino Unido Coombes y Jones (2016) con su proyecto "Beat The Street", donde de forma gamificada aquellos alumnos y colegios que recorrían más kilómetros sumaban mayor puntuación para ganar premios. También han funcionado proyectos que fomentan la cooperación y gamificación (Cerro-Herrero et al., 2021; LópezCenteno et al., 2021).

\section{Conclusiones}

La investigación desarrollada en este estudio plantea en primera instancia la falta de relación entre la intención de ser físicamente activo y el desplazamiento activo, lo cual debe llevar a reflexionar sobre las futuras intervenciones que pretendan potenciar el desplazamiento activo. Los escolares españoles suelen mostrar altos niveles de intención de ser físicamente activos y esto podría ser aprovechado para lograr mejores niveles de desplazamiento caminando o en bicicleta al colegio, pero para ello son necesarias intervenciones que transformen el desplazamiento activo en una experiencia más relacionada con la actividad física y no solo con una actividad cotidiana para movernos de un sitio a otro. Para ello se debe apostar por medidas que fomenten la competición, la gamificación, la cooperación, que supongan retos y que den visibilidad a los que desarrollan estas actividades (tabla 4). La lejanía al centro educativo es una de las principales barreras para que los niños y jóvenes realicen desplazamiento activo, pero con intervenciones adecuadas, puede ser transformada en un valor ya que puede permitir superar mayores retos, logras mayores puntuaciones y, en definitiva, realizar más actividad física. Para ello, los docentes e investigadores deben dar valor a la distancia recorrida en todas sus intervenciones para pasar este elemento de barrera al desplazamiento a motivación extra por realizar mayores distancias. 
Tabla 4.

Propuesta de valores a integrar en programas de fomento del desplazamiento activo.

\begin{tabular}{|c|c|c|}
\hline Valor & Justificación & $\begin{array}{r}\text { Ejemplo } \\
\end{array}$ \\
\hline Competición & $\begin{array}{l}\text { La motivación por realizar la } \\
\text { actividad se verá incrementada al } \\
\text { tener un reto que conseguir y poder } \\
\text { compararse con otros compañeros. }\end{array}$ & $\begin{array}{l}\text { "Beat de Street" proyecto en el cual cada } \\
\text { alumno recibía una tarjeta que debía sellar } \\
\text { cada día en varias de las } 40 \text { máquinas } \\
\text { distribuidas por varios barrios, y así } \\
\text { sumar puntos y compararse con los } \\
\text { compañeros (Coombes y Jones, 2016). }\end{array}$ \\
\hline Gamificación & $\begin{array}{l}\text { Incluir programas con puntuaciones, } \\
\text { superación de niveles puede } \\
\text { aumentar la motivación hacia el } \\
\text { desplazamiento activo. }\end{array}$ & $\begin{array}{l}\text { "One Step Forward" desarrollaron una } \\
\text { metodología para diseñar intervenciones } \\
\text { que fomenten el desplazamiento activo y } \\
\text { la gamificación es uno de sus principios } \\
\text { básicos (Lindqvist y Rutberg, 2018). }\end{array}$ \\
\hline Cooperación & $\begin{array}{l}\text { Plantear desafíos físicos } \\
\text { cooperativos relacionados con el } \\
\text { desplazamiento activo puede ser una } \\
\text { estrategia para fomentar la } \\
\text { cooperación grupal y hacer que el } \\
\text { desplazamiento activo pase a ser una } \\
\text { actividad grupal. }\end{array}$ & $\begin{array}{l}\text { "Desafíos físicos cooperativos" es una } \\
\text { propuesta de retos para educación física, } \\
\text { cuyos principios básicos podrían ser } \\
\text { adaptados para diseño de desafíos } \\
\text { relacionados con el desplazamiento } \\
\text { activo (Fernández-Río y Callado, 2005). }\end{array}$ \\
\hline Retos-objetivos & $\begin{array}{l}\text { Los participantes deben poder } \\
\text { marcarse objetivos o retos y poder ir } \\
\text { cuantificando su avance para } \\
\text { lograrlos. }\end{array}$ & $\begin{array}{l}\text { "Active Lions" pusieron en marcha una } \\
\text { aplicación en un campus universitario que } \\
\text { permitía a cada usuario planificar su } \\
\text { desplazamiento activo e ir cuantificando } \\
\text { lo realizado y lograr los objetivos o retos } \\
\text { que se marcaba cada individuo (Bopp et } \\
\text { al., 2018). }\end{array}$ \\
\hline Visibilidad & $\begin{array}{l}\text { La práctica deportiva suele dar cierta } \\
\text { repercusión positiva a aquellos que } \\
\text { la práctica por sus éxitos y los } \\
\text { valores que se le asocian, pero esto } \\
\text { no sucede con el desplazamiento } \\
\text { activo. }\end{array}$ & $\begin{array}{l}\text { "Playing in Traffic" pusieron en marcha } \\
\text { la grabación de los trayectos y su } \\
\text { publicación en una web, dando de este } \\
\text { modo visibilidad a la forma de } \\
\text { desplazarse y las experiencias vividas. } \\
\text { (Chan, 2011) }\end{array}$ \\
\hline
\end{tabular}

Es importante considerar los hallazgos encontrados con cautela. El presente trabajo muestra algunas limitaciones, como es la naturaleza transversal de estudio, la cual no permite establecer relaciones de causa-efecto. Además de considerar que solo se han analizado dos centros educativos en la misma ciudad, sería interesante ampliar el estudio en entornos urbanos y rurales.

Contribución de cada Autor: Conceptualización, C-H. D y T-S. M. A.; metodología, V-S. M y S-M. P. A.; validación, C-H. D, P-P. J y S-M. P. A; análisis, T-S. M. A; escritura del manuscrito, C-H. D; escritura, revisión y edición, T-S. M. A.; supervisión, P-P. J y S-M. P. A".

Financiación: Esta investigación ha sido financiada por FEDER, FSE y Gobierno de Extremadura, números de subvención GR18102 y TA18027.

Agradecimientos: Agradecemos el apoyo financiero de la Consejería de Economía e Infraestructuras y de la Comunidad Europea.

Conflicto de Intereses: Las/os autoras/es declaran que no tienen conflicto de intereses.

\section{Referencias}

Arias, J. L., Castejón, F. J., y Yuste, J. L. (2013). Propiedades psicométricas de la escala de intencionalidad de ser físicamente activo en educación primaria. Revista de Educación, 362, 485-505. https://doi.org/10.4438/1988-592X-RE-2013-362-239

Bandura, A. (2004). Health promotion by social cognitive means. Health Education and Behavior, 31, 143-164. https://doi.org/10.1177/1090198104263660

Espiral. Cuadernos del Profesorado | ISSN 1988-7701 | 2022, 15(30), 51-58 
Bopp, M., Sims, D., Matthews, S. A., Rovniak, L. S., Poole, E., y Colgan, J. (2018). Development, Implementation, and Evaluation of Active Lions: A Campaign to Promote Active Travel to a University Campus. American Journal of Health Promotion, 32(3), 536-545.

Cerro, D., Tapia, M., Vaquero, M., Prieto, J., \& Sánchez, P. (2021). Revisión sistemática sobre los beneficios psicosociales obtenidos con intervenciones para promover el desplazamiento activo al colegio. SPORT TKRevista EuroAmericana de Ciencias del Deporte, 10(1), 95-105. https://doi.org/10.6018/sportk.461711

Cerro, D., Vaquero, M., Prieto, J., Sánchez-Miguel, P. A., y Tapia, M. A. (2020). ¿Por qué los jóvenes cacereños no son más activos en su desplazamiento al centro educativo? Estudio piloto sobre barreras para el desplazamiento activo. Cuadernos de Investigación en Juventud, 8, 22-35.

Cerro-Herrero, D. et al. (2021). Diseño de juego de mesa para fomentar el desplazamiento activo al colegio entre los escolares: El camino al cole. Revista Española de Educación Física y Deportes, 432, 35-46

Coombes, E., \& Jones, A. (2016). Gamification of active travel to school: a pilot evaluation of the beat the street physical activity intervention. Health \& Place, 39, 62-69.

De la Cruz Bazaga, B. et al. (2021). Propuesta de intervención para fomentar el desplazamiento activo al centro educativo. Revista Española de Educación Física y Deportes, 432, 77-90

Chan, K. (2011). Visual ethnography in game design: A case study of user-centric concept for a mobile social traffic game. In Proceedings of the 15th International Academic MindTrek Conference: Envisioning Future Media Environments, MindTrek 2011. https://doi.org/10.1145/2181037.2181051

Chillón, P., Herrador-Colmenero, M., Migueles, J. H., Cabanas-Sánchez, V., Fernández-Santos, J. R., Veiga, Ó. L., ... Gómez-Gallego, F. (2017). Convergent validation of a questionnaire to assess the mode and frequency of commuting to and from school. Scandinavian Journal of Public Health, 45(6), 612-620. https://doi.org/10.1177/1403494817718905

Coombes, E., y Jones, A. (2016). Gamification of active travel to school: A pilot evaluation of the Beat the Street physical activity intervention. Health \& Place, 39, 62-69. https://doi.org/10.1016/j.healthplace.2016.03.001

Escobar-Gómez, D., Rodríguez-Rodríguez, F., Villa-González, E., Esteban-Cornejo, I., y Chillón-Garzón, P. (2020). Fiabilidad y viabilidad de un cuestionario autorreportado sobre el modo, tiempo y distancia de desplazamiento en niños y adolescentes. Retos: Nuevas Tendencias En Educación Física, Deporte y Recreación, 37(1579-1726), 379-385. https://recyt.fecyt.es/index.php/retos/article/view/71639

Fernández-Río, J., y Callado, C. V. (2005). Desafios físicos cooperativos: retos sin competición para las clases de Educación Física. Wanceulen.

García-Hermoso, A., Saavedra, J. M., Olloquequi, J., y Ramírez-Vélez, R. (2017). Associations between the duration of active commuting to school and academic achievement in rural Chilean adolescents. Environmental Health and Preventive Medicine, 22(31), 1-7. https://doi.org/10.1186/s12199-017-0628-5

Garrote-García, J. (2016). Estudio del nivel de actividad física y su vinculación con el rendimiento académico y el desplazamiento activo al centro educativo en alumnos de Secundaria. Universidad de León. https://bit.ly/3Fqs5ZS

Hein, V., Müür, M., y Koka, A. (2004). Intention to be Physically Active after School Graduation and Its Relationship to Three Types of Intrinsic Motivation. European Physical Education Review, 10, 5-19. https://doi.org/10.1177/1356336x04040618

Henriques-Neto, D., Peralta, M., Garradas, S., Pelegrini, A., Pinto, A. A., Sánchez-Miguel, P. A., y Marques, A. (2020). Active Commuting and Physical Fitness: A Systematic Review. International Journal of Environmental Research and Public Health, 17(2721). https://doi.org/10.3390/ijerph17082721

Herrador-Colmenero, M. Escabias, M., Ortega, F. B., McDonald, N. C., y Chillón, P. (2019). Mode of Commuting TO and FROM School: A Similar or Different Pattern? Sustainability, 11(4), 1026. https://doi.org/https://doi.org/10.3390/su11041026

Kamtsios, S. (2011). Differences in attitudes towards exercise, perceived athletic ability, perceived physical attractiveness and participation in physical activity in children and adolescents aged 10 to 18 years old. Journal of Sport and Health Research, 3(3), 129-142.

Larouche, R., Saunders, T. J., Faulkner, G. E. J., Colley, R., y Tremblay, M. (2014). Associations between active school transport and physical activity, body composition, and cardiovascular fitness: A systematic review of 68 studies. Journal of Physical Activity and Health, 11(1), 206-227 https://doi.org/10.1123/jpah.20110345 
Lee, M. C., Orenstein, M. R., y Richardson, M. J. (2008). Systematic review of active commuting to school and children's physical activity and weight. Journal of Physical Activity and Health, 5(6), 930-949. https://doi.org/10.1123/jpah.5.6.930

Lindqvist, A. K., y Rutberg, S. (2018). One step forward: Development of a program promoting active school transportation. Journal of Medical Internet Research, 7(5), e123. https://doi.org/10.2196/resprot.9505

López-Centeno, F. D.; Gálvez-Fernández, P.; Herrador-Colmenero, M.; Lara-Sánchez, A. J. (2021). Intervención educativa para incertivar hábitos de desplazamiento active al colegio en escolares de Primaria. Journal of Sport and Health Research, 13(2), 331-346

Mann, M., Silver, E. J., y Stein, R. E. K. (2018). Active Commuting to School, Physical Activity, and Behavior Problems Among Third-Grade Children. Journal of School Health, 88(10), 734-737. https://doi.org/10.1111/josh.12677

Martínez-Baena, A. C. (2016). Niveles de actividad y condición física saludable en escolares de educación secundaria obligatoria: implicaciones para el profesorado de EF. Universidad de Granada.

Mayorga-Vega, D., Parra-Saldías, y Viciana, J. (2019). Condición física, actividad física, conducta sedentaria y predictores psicológicos en adolescentes chilenos: diferencias por género. Cultura, Ciencia y Deporte, 14(42), 233-241. https://doi.org/http://dx.doi.org/10.12800/ccd.v14i42.1337

Molina-García, J., Queralt, A., Estevan, I., Álvarez, O., y Castillo, I. (2016). Barreras percibidas en el desplazamiento activo al centro educativo: fiabilidad y validez de una escala. Gaceta Sanitaria, 30(6), 426-431. https://doi.org/10.1016/j.gaceta.2016.05.006

Moreno, J. A., Moreno, R., y Cervelló, E. (2007). El autoconcepto físico como predictor de la intención de ser físicamente activo. Psicología y Salud, 17(2), 261-267. https://doi.org/10.25009/pys.v17i2.710

Pérez-Soto, J. J., García-Cantó, E., Rosa-Guillamón, A., Rodríguez-García, P. L., Moral-García, J. E., y LópezGarcía, S. (2019). Relación entre la intención de ser activo y la actividad física extraescolar. Revista de Psicología, 37(1)(0254-9247), 389-405. https://doi.org/10.18800/psico.201902.001

Rodríguez-López, J. P. (2017). Patrones y determinantes del desplazamiento activo al centro escolar en niños y adolescentes españoles. Universidad de Granada. http://hdl.handle.net/10481/48130

Rosa-Guillamón, A. (2019). Análisis de la relación entre salud, ejercicio físico y condición física en escolares y adolescentes. Revista Ciencias de la Actividad Física UCM, 20(1), 1-14. https://doi.org/http://doi.org/10.29035/rcaf.20.1.1

Ruiz-Pérez, L. M., Ramón-Otero, I., Palomo-Nieto, M., Ruiz-Amengual, A., y Navia-Manzano, J. A. (2014). La Intención de Practicar en el Futuro en Escolares Adolescentes. Kronos, 13(2). https://bit.ly/32pNDXW

Sanz, E., Elizondo, A., y Fraguela, R. (2017). Desplazamiento activo de los adolescentes al centro de estudios y funcionamiento familiar. Apunts. Educación Física y Deportes, 128, 36-47. https://doi.org/10.5672/apunts.2014-0983.es.(2017/2).128.02

To, Q. G., Gallegos, D., Do, D. V., Tran, H. T., To, K. G., Wharton, L., y Trost, S. G. (2020). Correlates of physical activity in fifth-grade students in Ho Chi Minh City, Vietnam. Sports Medicine and Health Science, 2, $33-$ 37. https://doi.org/10.1016/j.smhs.2020.02.002 